\title{
Real-time intrinsic fluorescence visualisation and sizing of proteins and protein complexes in microfluidic devices
}

\author{
Pavan Kumar Challa, ${ }^{\dagger, \perp}$ Quentin Peter, ${ }^{\dagger, \perp}$ Maya A. Wright, ${ }^{\dagger, \ddagger}$ Yuewen Zhang, ${ }^{\dagger}$ \\ Kadi L. Saar, ${ }^{\dagger}$ Jacqueline A. Carozza, ${ }^{\dagger} \uparrow$ Justin L.P. Benesch, ${ }^{\S}$ and Tuomas P. J.
} Knowles ${ }^{*, \dagger}, \|$

$\dagger$ Department of Chemistry, University of Cambridge, Lensfield Road, CB2 1EW,

1

$\ddagger$ Current address : Fluidic Analytics, Unit 5 Chesterton Mill, French’s Road, Cambridge CB4 3NP, UK

\Current address : Department of Chemistry, Stanford University, Beckman Center, 279 W. Campus Drive, Stanford, California, 94305, USA.

$\S$ Department of Chemistry, Physical \&3 Theoretical Chemistry Laboratory, University of Oxford, South Parks Road, Oxford, Oxfordshire, OX1 3QZ, UK.

॥Cavendish Laboratory, University of Cambridge, J J Thomson Avenue, CB3 1HE, \author{
Cambridge, UK. \\ $\perp$ These authors contributed equally to this work. \\ E-mail: tpjk2@cam.ac.uk
}

\begin{abstract}
Optical detection has become a convenient and scalable approach to read out information from microfluidic systems. For the study of many key biomolecules, however,
\end{abstract}


including peptides and proteins, which have low fluorescence emission efficiencies at visible wavelengths, this approach typically requires labelling of the species of interest with extrinsic fluorophores to enhance the optical signal obtained - a process which can be time-consuming, requires purification steps, and has the propensity to perturb the behaviour of the systems under study due to interactions between the labels and the analyte molecules. As such, the exploitation of the intrinsic fluorescence of protein molecules in the UV range of the electromagnetic spectrum is an attractive path to allow the study of unlabelled proteins. However, direct visualisation using $280 \mathrm{~nm}$ excitation in microfluidic devices has to date commonly required the use of coherent sources with frequency multipliers and devices fabricated out of materials that are incompatible with soft-lithography techniques. Here, we have developed a simple, robust and cost-effective $280 \mathrm{~nm}$ LED platform that allows real-time visualisation of intrinsic fluorescence from both unlabelled proteins and protein complexes in polydimethylsiloxane microfluidic channels fabricated through soft-lithography. Using this platform, we demonstrate intrinsic fluorescence visualisation of proteins at nanomolar concentrations on chip, and combine visualisation with micron-scale diffusional sizing to measure the hydrodynamic radii of individual proteins and protein complexes under their native conditions in solution in a label-free manner.

\section{Introduction}

Proteins underpin most of the key functional processes in cells, and there has thus been a sustained and long-standing interest in developing tools capable of studying proteins under native conditions in solution. Microfluidic platforms are highly attractive in the context of protein science; they minimise sample consumption, cost, and measurement time. Moreover, exploitation of laminar fluid flow to integrate multiple functions onto a compact microfluidic chip platform enables miniaturisation, thus leading to the ability to readily perform studies that are impractical in conventional bulk studies. These characteristics lead to the potential 
of such systems to impact fields ranging from medical diagnostics, genetic analysis and drug discovery to proteomics. Applications including DNA sequencing, polymerase chain reaction, capillary electrophoresis, DNA separation, enzymatic assays, immunoassay's, cell counting, cell sorting, and cell culture have been successfully miniaturised onto a chip. ${ }^{1-5}$

A variety of methods have been developed to obtain information about the conformational states and folding pathways of biomolecules in solution, most notably these of proteins. ${ }^{6-11}$ Among these methods, fluorescence spectroscopy, where changes in the fluorescence intensity at a fixed wavelength or shifts in the wavelength at maximum intensity are observed, has been used successfully to study conformational changes of proteins due to the high sensitivity of this approach. ${ }^{12}$ Generally for such biophysical characterisation, protein molecules are studied with extrinsic labels due to the high signal-to-noise ratios that they afford. Despite the fact that there have been significant technological developments in the photochemistry and photophysics of modern dye molecules, labelling remains inherently a time-consuming and labour intensive process. Furthermore, extrinsic labelling has the propensity to perturb the folding processes and kinetics of proteins. ${ }^{12-14}$ Hence, label free techniques have in principle great advantages for studying protein conformations and can play a key role in other numerous bio-detection applications. ${ }^{15}$ Typically, when excited with UV light below 300nm proteins exhibit intrinsic fluorescence from the aromatic amino acids tryptophan, tyrosine, and phenylalanine. ${ }^{16}$

As such, label-free techniques have clear inherent advantages over label-based ones, particularly in the context of the study of protein-protein interactions which can be perturbed by the labels. However, integration of intrinsic fluorescence visualisation with lab on a chip platforms has remained challenging due to the inherently low sensitivity confounded by the constraint of limited optical path lengths in reduced volumes. Nevertheless, even though visualisation applications have remained challenging, detection through UV-absorptionbased measurements of native proteins in microfluidic chips have been demonstrated successfully. ${ }^{17-20}$ Due to the limited path lengths achievable in microfluidic devices, high levels 
of sensitivity remain challenging to achieve. To overcome these limitations several groups have used laser-based technologies through frequency doubled, tripled or quadrupled continuous wave and pulsed lasers for native fluorescence detection of proteins in a fused silica and PDMS microchips using a photomultiplier tube detectors. ${ }^{20-25}$ In particular, high speed laser scanners with a frequency quadrupled laser for deep UV fluorescence detection and visualisation of proteins has been demonstrated in a free flow electrophoresis fused silica chip. ${ }^{21}$ Moreover, the frequency tripled output of Ti:Sapphire laser has been integrated to a fused silica microchip to study quenching and refolding kinetics in a laser machined flow mixer device using time correlated single photon counting(TCSPC) fluorescence detection system. ${ }^{26}$ In addition, frequency quadrupled Nd:YAG lasers (266 nm) have been exploited to explore protein separation and detection. ${ }^{27}$ In order to alleviate the complexity of frequency multiplying approaches conventionally required for generating high power coherent radiation at $280 \mathrm{~nm}$ non-coherent sources, including $230 \mathrm{~nm}$ high intensity deuterium lamps ${ }^{28}$ or more recently UV-LED at $280 \mathrm{~nm}$ have been used to perform detection in capillary electrophoresis of native proteins using photomultiplier tubes. ${ }^{29,30}$ These systems represent significant advances as they allow unlabelled proteins to be studied in microfluidic systems, but in cases where high sensitivity is required such approaches have relied on materials and devices which are not compatible with rapid soft lithography fabrication techniques. Light emitting diodes(LED's) are simple, stable, cost effective, have long life-times, and a small size. However, the low output power of the LEDs has hindered their use as a light source for intrinsic fluorescence visualisation of proteins in real time flowing through PDMS microfluidic devices using charge coupled device (CCD) cameras.

Here we have designed and implemented a compact 280nm high power LED microchip based fluorescence visualisation platform which is fully compatible with rapid soft lithography microfabrication approaches, opening up the possibility of using label free protein studies in a wide range of device designs and architectures. Excitation at $280 \mathrm{~nm}$ enables visualisation of proteins molecules via the autofluorescence of their tryptophan and tyrosine 
amino acid residues. To illustrate the power of this method, we combine autofluorescence detection with diffusional sizing to measure the hydrodynamic radius of monomeric proteins bovine serum albumin (BSA), lysozyme and oligomeric clusters of the molecular chaperone $\alpha$ B-crystallin in free solution in a label free manner by following their mass transport in real time in microchannels.

\section{Experimental Details}

\section{Intrinsic fluorescence visualisation platform}

We built a robust and compact $280 \mathrm{~nm}$-LED based epifluorescence microfluidic station for label free fluorescence visualisation of proteins on chip, using fluorescence from the aromatic amino acids tryptophan and tyrosine (figure 1). Light from a $280 \mathrm{~nm}$ LED (Thorlabs M280L3) is passed through an aspherical lens of focal length $20 \mathrm{~mm}$ to obtain a nearly collimated beam. This beam is incident on a dichroic filter cube, which consists of an excitation filter (Semrock FF01-280/20-25) centred at a wavelength $280 \mathrm{~nm}$, and a dichroic mirror (Semrock FF310-Di01-25x36). The light is again reflected by a UV-enhanced aluminium mirror (Thorlabs CCM1-F01/M) and focused onto the sample flowing in a microfluidic device by an infinity corrected UV objective lens (magnification 10X, numerical aperture $=$ 0.25). The fluorescence from the sample is collected through the same objective and passed through an emission filter (Semrock FF01-357/44-25) centred at a wavelength of $357 \mathrm{~nm}$, and finally focused onto a EMCCD camera (Rolera EM-C2) by an air-spaced achromatic doublet lens (Thorlabs ACA254-200-UV) of focal length $200 \mathrm{~mm}$. The exposure time used in our experiments was about 500ms.

\section{Device Fabrication}

Microfluidic devices for intrinsic fluorescence visualisation experiments are cast using polydimethylsiloxane (PDMS) (Sylgard 184 kit, Dow Corning) from a silicon wafer master im- 
printed with $50 \mu \mathrm{m}$ high channels fabricated using conventional UV lithography. ${ }^{31,32}$ Carbon black nano-powder (Sigma-Aldrich) is added to the PMDS before curing to create black devices, thus minimising unwanted autofluorescence from PDMS under 280nm-LED illumination during the measurements. Devices are bonded to a quartz slide (Alfa Aesar, $76.2 \mathrm{x}$ $25.4 \times 1.0 \mathrm{~mm}$ ) using a plasma bonder (Electronic Diener Femto, $40 \%$ power for 15s) and subsequently plasma treated for $500 \mathrm{~s}$ to render the channels hydrophilic. The channels are filled from the outlet with buffer using a glass syringe (Hamilton, $500 \mu \mathrm{L}$ ), equipped with a needle (Neolus Terumo, 25 gauge, $0.5 \times 16 \mathrm{~mm}$ ), and polyethene tubing (Scientific Laboratory Supplies, inner diameter $0.38 \mathrm{~mm}$, outer diameter $1.09 \mathrm{~mm}$ ). The microfluidic devices used in this study are the diffusional sizing devices described in. ${ }^{33,34}$

\section{Background Correction}

An autofluorescence image of a protein sample in a microfluidic device taken on the deep UV set-up can be separated into three contributions: the signal from the protein, the signal from the background, and noise. A better signal-to-noise ratio can be obtained by generating a larger volume of statistically independent data, for example through longer exposure times and by acquiring series of pictures, or by decreasing the resolution by binning and Gaussian filtering, approaches which both reduce statistical noise. The background signal, by contrast, is constant across images and can thus be removed by comparing an image with and without sample present. Here, we implement this process in three steps (figure 2). First, the nonuniform illumination distribution is extracted from the outside of the channel, which is expected to be flat on the large scale despite local variations due to the carbon nano-powder. This distribution, obtained with fitting a second order 2D polynomial (figure 2b), is then divided from the image. Second, the two images (with and without fluorescence signal from proteins) are registered. They might present a relative translation, rotation or scale difference, which are corrected through analysis in Fourier space: ${ }^{35}$ given a function $f_{2}$ that 
is a translation of a function $f_{1}$, the Fourier transforms $F_{1}$ and $F_{2}$ are related by:

$$
\begin{aligned}
& f_{2}(x, y)=f_{1}\left(x-x_{0}, y-y_{0}\right) \\
& F_{2}(\xi, \eta)=e^{-2 \pi i\left(\xi x_{0}+\eta y_{0}\right)} F_{1}(\xi, \eta)
\end{aligned}
$$

The phase term can be isolated. Using $\left\|F_{1}\right\|=\left\|F_{2}\right\|$ :

$$
e^{-2 \pi i\left(\xi x_{0}+\eta y_{0}\right)}=\frac{F_{2}(\xi, \eta) F_{1}^{*}(\xi, \eta)}{\left\|F_{2}(\xi, \eta) F_{1}(\xi, \eta)\right\|}
$$

$x_{0}$ and $y_{0}$ are found by taking the Fourier transform of Eq. (2). Given $f_{2}$ a rotated and rescaled version of $f_{1}$, a change of coordinates to log-polar yields a translation of the form:

$$
\begin{aligned}
f_{2}(x, y) & =f_{1}\left(x / a \cdot \cos \theta_{0}+y / a \cdot \sin \theta_{0}, \ldots\right) \\
f_{2}(\log \rho, \theta) & =f_{1}\left(\log \rho-\log a, \theta-\theta_{0}\right)
\end{aligned}
$$

The angle and scale difference can therefore be found by using the same method. Eq. (1) shows that the magnitude of the Fourier transform does not depend on translation, and can therefore be used to find the relative angle and scaling. The offset can be found after rotation and scaling. The logarithm of the log-polar representation of the magnitude is used to find the angle and scale to avoid over-attributing importance to large-scale features. ${ }^{35}$ Finally, the signal and background images are subtracted. The units of the resulting image are a ratio between the signal and the background amplitude. This is conserved between different lamp intensities, but not between devices. Therefore, a calibration step for each new device is necessary.

\section{Protein samples}

Bovine serum albumin (BSA) was purchased from Sigma-Aldrich (product number \#A9418; lyopholised powder used without further purification) and dilutions prepared in $25 \mathrm{mM}$ sodium phoshpate buffer pH 8.0. Chicken lysozyme (Sigma-Aldrich L6876; lyophilised pow- 
der used without further purification) was dissolved in $2.5 \mathrm{mM}$ phosphate buffer at $\mathrm{pH} 8.0$ to a final concentration of $200 \mu \mathrm{M}$ with the concentrations similarly determined by NanoDrop spectrophotometer. $560 \mu \mathrm{M} \alpha$-synuclein solutions were prepared in $25 \mathrm{mM}$ phosphate solution buffer ( $\mathrm{pH}$ 7.4). ${ }^{36} 120 \mu \mathrm{M} \alpha \mathrm{B}$-crystallin solutions were prepared in $2.5 \mathrm{mM}$ phosphate buffer (pH 8).

\section{Results and Discussion}

Visualisation of low intensity autofluorescence from proteins using excitation with $280 \mathrm{~nm}$ radiation poses challenges for the commonly used materials in soft lithography, including PDMS and glass, which absorb most of this light and exhibit significant background fluorescence, which decreases the imaging contrast. In order to address this challenge, a number of technical steps are required. First, we selected all optical components, including the microscope slide to which the microfluidic device is bonded, from quartz to avoid absorption by NBK7 glass. In addition, black carbon nano-powder was mixed with the PDMS during device fabrication to minimise unwanted autofluorescence from the PDMS. ${ }^{25}$ Since statistical noise is proportional to the square root of the signal amplitude, a large background signal produces a large associated noise that decreases the signal-to-noise ratio dramatically. Moreover, although the presence of the black carbon nano-powder reduces the overall background, it introduces spatial irregularities in the measured signal. This can be observed in figure $\mathbf{1}(\mathbf{c}-\mathbf{d})$. We addressed this limitation with an image processing approach that included a specifically incorporated background subtraction (figure 2). These three steps can be used with almost any PDMS microfluidics device design to allow analysis of unlabelled proteins that previously needed to be labelled with extrinsic fluorophores. By using black devices bonded to quartz and subjecting the images to our processing steps, we improve the signal-to-noise ratio by a factor of 10 over imaging in a conventional clear PDMS device bonded to glass (figure 2). 
To test if our approach was sufficiently sensitive to be applied to study proteins, we chose a representative set of proteins, which included BSA(figure 3), lysozyme(figure 1(c)) and $\alpha \mathrm{B}$-crystallin, which contain tryptophan residues and $\alpha$-synuclein(figure $1(\mathrm{~d})$ ), which does not contain any tryptophan residues, but has 4 tyrosine residues. Our results are in figures 1(d) show that we could certainly visualise the autofluorescence from $\alpha$-synuclein.

\section{Detection limit}

Having established the principles for operating the $280 \mathrm{~nm}$-LED microfluidic platform, we explored the limits of visualisation afforded through this approach. Our data show that for BSA, the lowest concentration where the signal-to-noise ratio is large enough to image a profile at position 0 in the device design shown in figure 3(a) is $100 \mathrm{nM}$ (figure 4). The lowest concentration of BSA that is visible at all positions, thus allowing for label-free sizing, is $500 \mathrm{nM}$, the amplitude at position 12 being approximatively five times less than position 0 , as seen in higher concentration measurements (figure 3c). The hydrodynamic radius of BSA measured through this approach is $3.3 \pm 0.6 \mathrm{~nm}$, which is close to the literature value determined at higher concentrations ${ }^{14,37}$ in bulk systems or by using labelling approaches.

We can estimate the sensitivity of our platform for a generic protein that has tryptophan and tyrosine amino acids as follows. The average abundance of tryptophan in the human proteome is c.a $1.3 \%^{38,39}$ and apparently, the average sequence is c.a 480 amino acids, which means that an average protein has $480 * 0.013=6$ tryptophan residues. The data is shown in figure 4 for $100 \mathrm{nM}$ BSA corresponds to $300 \mathrm{nM}$ of tryptophan residues; taking this value as the detection limit, we obtain the concentration that can be measured for an average protein to be c.a $300 \mathrm{nM} / 6=50 \mathrm{nM}$. A similar argument can be applied to goes for tyrosine; the average abundance in this case is around $3.3 \%^{38,39}$ so for an average protein there are approximately $480 * 0.033=15$ tyrosine residues. As such, for an average protein the detection limit from the signal of tyrosine 
residues alone is $2.1 \mu \mathrm{M} / 15=140 \mathrm{nM}$.

\section{Microfluidic diffusional sizing with fluorescence visualisation}

To demonstrate the potential of our $280 \mathrm{~nm}$-LED fluorescence visualisation platform, we used the microfluidic device shown in figure 3 a to monitor the micron-scale diffusive mass transport of native proteins in space and time to determine their hydrodynamic radii. ${ }^{14,33,34}$ To this effect, 12 images are taken along a $100 \mathrm{~mm}$ diffusion channel and processed into a set of lateral scan profiles, which are then fitted to a set of simulated basis functions. ${ }^{33}$ Information about the spatial diffusion, transverse to the flow, and temporal diffusion, along the advective direction is obtained from the diffusion profiles by deconvolving the experimental profiles into a linear combination of profiles expected for particles with known diffusion coefficients. A least-squares error algorithm is used to find the linear combination yielding the lowest residuals, allowing the average radius of the analyte to be determined. ${ }^{33,34}$ We first measured the hydrodynamic radii of bovine serum albumin (BSA), a transport protein with 583 amino acids ( 66,500 Da), present in blood plasma, and chicken lysozyme - an antimicrobial enzyme that forms part of the innate immune system. The results shown in figure 3 and 4 show that, using this approach, we are able to visualise directly spatio-temporal distribution of unlabelled BSA on chip. Figure 3b shows typical microscopy images of diffused $15 \mu \mathrm{M} \mathrm{BSA}$ at different positions along the microfluidic channels.

\section{Fluorescence visualisation and sizing of protein complexes}

We next focused on $\alpha \mathrm{B}$-crystallin $(\alpha \mathrm{B}-\mathrm{C})$ which is a 175 amino acid long polypeptide chain with molecular mass of $20.1 \mathrm{kDa}$. Our results show that the measured hydrodynamic radius for this system is significantly larger than that expected from a scaling relationship between molecular mass and radius, (figure 5). These findings obtained under fully native conditions and for unlabelled molecules, indicates that the monomeric protein is forming complexes under these conditions. Sizing of self-assembled protein-structures can be challenging with 
many conventional techniques as such non-covalent complexes are held together via weak interactions that have the propensity to be altered as soon as the proteins are moved away from under native conditions. The present results therefore open up the possibility of studying not only individual protein molecules, but nanoscale protein complexes under fully native conditions in an entirely label free manner.

These results on the size of $\alpha \mathrm{B}$-crystallin complexes under native conditions allow us to access an estimate of the number of monomer units self-assembled into a cluster. To this effect, we combined the hydrodynamic radii of proteins from reference ${ }^{40,41}$ with our data and plotted as function of their molecular mass (figure 5). We then fit the data using the formula, $M_{w}=R_{h}^{3} / \alpha$ where $R_{h}$ is the hydrodynamic radius of the proteins, $\alpha$ is the scaling coefficient, and $M_{w}$ is the molecular mass. Then, using the fitted values $\alpha=0.083749 \pm$ $0.00175 \mathrm{~nm}^{3} / \mathrm{Da}$ and measured hydrodynamic radius $R_{h}=6.69 \mathrm{~nm} \pm 0.63$, we estimated the overall molecular mass of the cluster to be $510 \pm 148 k D a$ and thus the aggregation number to be $510 \pm 148 k D a / 20.1 k D a=25.4 \pm 7.3$. This value, measured under native conditions in free solution, is consistent with other measurements performed both in the solution and the gas phase in which $\alpha$ B-crystallin has been observed to form polyhedral oligomers with sizes ranging from 10-mers to 40-mers, with the species of highest abundance in the range between 24-32 subunits. ${ }^{42}$ These results are thus in good agreement with biophysical characterisation performed in the gas phase using native mass spectrometry experiments. ${ }^{43,44}$ We thereby demonstrate that the intrinsic fluorescence set-up developed here is a powerful tool for the analysis of key biomolecules of physiological importance, and moreover, can be coupled with microfluidics to observe the self-assembly phenomena of proteins under native conditions in free-solution. 


\section{Conclusions}

Analytical tools for characterising proteins and their complexes in solution phase without extrinsic labels are actively sought after for molecular biology and structural biology applications. The intrinsic fluorescence from proteins originates mainly from the aromatic residues tryptophan and tyrosine. We have described, designed and built a novel $280 \mathrm{~nm}-$ LED based fluorescence visualisation platform for characterising unlabelled proteins, at nanomolar concentrations in the solution phase, within microfluidic devices fabricated using soft-lithography. This platform allowed us to visualise in real time the spatial distribution on the micron scale of intrinsic fluorescence of nanoscale proteins and protein complexes within microfluidic channels. Our results highlight the potential of this approach for label-free fluorescenceand size measurements which consume small amounts of sample, have fast processing times, and is robust for large scale integration of multiple components on a single chip. As an illustration of the power of this approach, we combined fluorescence imaging with diffusional sizing on chip to measure the hydrodynamic radius of proteins and self-assembled protein clusters of biological interest under their native conditions. The ability to image unlabelled proteins in solution in PDMS microfluidic chips has the potential to enable further studies of protein folding and unfolding pathways, kinetics, protein-protein interactions and opens up the possibility of studying unlabelled proteins in a variety of microfluidic devices and architectures.

\section{Acknowledgement}

The research leading to these results has received funding from the European Research Council under the European Union's Seventh Framework Programme (FP7/2007-2013) through the ERC grant PhysProt (agreement $n^{\circ} 337969$ ). This project has received funding from the European Union's Horizon 2020 research and innovation programme under grant agreement No 674979-NANOTRANS. Moreover, we gratefully acknowledge the support of ERC with 
sponsor reference is 337969, EPSRC, BBSRC with sponsor reference is BB/J002119/1.

\section{Supporting Information Available}

The code used for correcting the background in figure 2 is accessible with the DOI https: //doi.org/10.5281/zenodo.1155526. The images are accessible with the DOI https: //doi.org/10.17863/CAM.18873. This material is available free of charge via the Internet at http://pubs.acs.org/.

\section{References}

(1) McDonald, J. C.; Whitesides, G. M. Accounts of Chemical Research 2002, 35, 491-499.

(2) Auroux, P.-A.; Iossifidis, D.; Reyes, D. R.; Manz, A. Analytical Chemistry 2002, 74, $2637-2652$.

(3) Buchholz, B. A.; Doherty, E. A. S.; Albarghouthi, M. N.; Bogdan, F. M.; Zahn, J. M.; Barron, A. E. Analytical Chemistry 2001, 73, 157-164.

(4) Kameoka, J.; Craighead, H. G.; Zhang, H.; Henion, J. Analytical Chemistry 2001, 73, $1935-1941$.

(5) Hatch, A.; Kamholz, A. E.; Hawkins, K. R.; Munson, M. S.; Schilling, E. A.; Weigl, B. H.; Yager, P. Nat Biotech 2001, 19, 461-465.

(6) Pelton, J. T.; McLean, L. R. Analytical Biochemistry 2000, 27\%, 167-176.

(7) Dyson, H. J.; Wright, P. E. Chemical reviews 2004, 104, 3607-22.

(8) Chiti, F.; Taddei, N.; van Nuland, N. A.; Magherini, F.; Stefani, M.; Ramponi, G.; Dobson, C. M. Journal of molecular biology 1998, 283, 893-903.

(9) Dousseau, F.; Pezolet, M. Biochemistry 1990, 29, 8771-8779. 
(10) Shalongo, W.; Heid, P.; Stellwagen, E. Biopolymers 1993, 33, 127-34.

(11) Kartanas, T.; Ostanin, V.; Challa, P. K.; Daly, R.; Charmet, J.; Knowles, T. P. Analytical Chemistry 2017, 89, 11929-11936, PMID: 28984439.

(12) Toseland, C. P. Journal of Chemical Biology 2013, 6, 85-95.

(13) Saar, K.-L.; Yates, E. V.; Müller, T.; Saunier, S.; Dobson, C. M.; Knowles, T. P. Biophysical Journal 2016, 110, 555 - 560.

(14) Yates, E. V.; Müller, T.; Rajah, L.; De Genst, E. J.; Arosio, P.; Linse, S.; Vendruscolo, M.; Dobson, C. M.; Knowles, T. P. J. Nat Chem 2015, 7, 802-809, Article.

(15) Emmelkamp, J.; Wolbers, F.; Andersson, H.; Dacosta, R. S.; Wilson, B. C.; Vermes, I.; van den Berg, A. Electrophoresis 2004, 25, 3740-5.

(16) Lakowicz, J. R., Ed. Principles of Fluorescence Spectroscopy; Springer US: Boston, MA, 2006; pp 529-575.

(17) Petersen, N. J.; Mogensen, K. B.; Kutter, J. P. ELECTROPHORESIS 2002, 23, 35283536.

(18) Liang, Z.; Chiem, N.; Ocvirk, G.; Tang, T.; Fluri, K.; Harrison, D. J. Analytical Chemistry 1996, 68, 1040-1046.

(19) Nakanishi, H.; Nishimoto, T.; Arai, A.; Abe, H.; Kanai, M.; Fujiyama, Y.; Yoshida, T. ELECTROPHORESIS 2001, 22, 230-234.

(20) Schulze, P.; Belder, D. Analytical and Bioanalytical Chemistry 2009, 393, 515-525.

(21) Köhler, S.; Nagl, S.; Fritzsche, S.; Belder, D. Lab Chip 2012, 12, 458-463.

(22) Quentmeier, S.; Denicke, S.; Gericke, K.-H. Journal of Fluorescence 2009, 19, 10371043. 
(23) Timperman, A. T.; Oldenburg, K. E.; Sweedler, J. V. Analytical Chemistry 1995, 67, $3421-3426$.

(24) Schulze, P.; Ludwig, M.; Belder, D. ELECTROPHORESIS 2008, 29, 4894-4899.

(25) Hellmich, W.; Greif, D.; Pelargus, C.; Anselmetti, D.; Ros, A. Journal of Chromatography $A$ 2006, 1130-195.

(26) Bilsel, O.; Kayatekin, C.; Wallace, L. A.; Matthews, C. R. Review of Scientific Instruments 2005, 76, 014302-014302.

(27) Hellmich, W.; Pelargus, C.; Leffhalm, K.; Ros, A.; Anselmetti, D. Electrophoresis 2005, 26, 3689-96.

(28) Zhu, L.; Lee, C. S.; DeVoe, D. L. Lab on a chip 2006, 6, 115-20.

(29) Sluszny, C.; He, Y.; Yeung, E. S. Electrophoresis 2005, 26, 4197-203.

(30) Tripathi, A.; Bozkurt, O.; Chauhan, A. Physics of Fluids 2005, 17, 103607-103607.

(31) Qin, D.; Xia, Y.; Whitesides, G. M. Nat. Protocols 2010, 5, 491-502.

(32) Challa, P. K.; Kartanas, T.; Charmet, J.; Knowles, T. P. J. Biomicrofluidics 2017, 11, 014113

(33) Müller, T.; Arosio, P.; Rajah, L.; Cohen, S. I. A.; Yates, E. V.; Vendruscolo, M.; Dobson, C. M.; Knowles, T. P. J. International Journal of Nonlinear Sciences and Numerical Simulation 2016, 0, 0.

(34) Arosio, P.; Müller, T.; Rajah, L.; Yates, E. V.; Aprile, F. A.; Zhang, Y.; Cohen, S. I. A.; White, D. A.; Herling, T. W.; De Genst, E. J.; Linse, S.; Vendruscolo, M.; Dobson, C. M.; Knowles, T. P. J. ACS Nano 2016, 10, 333-341, PMID: 26678709.

(35) Reddy, B. S.; Chatterji, B. N. IEEE TRANSACTIONS ON IMAGE PROCESSING 1996, VOL. 5, 1266-1271. 
(36) Hoyer, W.; Antony, T.; Cherny, D.; Heim, G.; Jovin, T. M.; Subramaniam, V. Journal of Molecular Biology 2002, 322, 383-393.

(37) Axelsson, I. Journal of Chromatography A 1978, 152, 21 - 32.

(38) King, J. L.; Jukes, T. H. Science 1969, 164, 788-798.

(39) Dyer, K. F. Journal of Biological Education 1971, 5, 15-24.

(40) Venturoli, D.; Rippe, B. American journal of physiology. Renal physiology 2005, 288, F605-13.

(41) Frigon, R. P.; Leypoldt, J. K.; Uyeji, S.; Henderson, L. W. Analytical Chemistry 1983, $55,1349-1354$.

(42) Hochberg, G. K. A.; Ecroyd, H.; Liu, C.; Cox, D.; Cascio, D.; Sawaya, M. R.; Collier, M. P.; Stroud, J.; Carver, J. A.; Baldwin, A. J.; Robinson, C. V.; Eisenberg, D. S.; Benesch, J. L. P.; Laganowsky, A. Proc. Natl. Acad. Sci. U. S. A. 2014, 111, E1562-70.

(43) Baldwin, A. J.; Lioe, H.; Robinson, C. V.; Kay, L. E.; Benesch, J. L. P. J. Mol. Biol. 2011, 413, 297-309.

(44) Aquilina, J. A.; Benesch, J. L. P.; Bateman, O. A.; Slingsby, C.; Robinson, C. V. Proc. Natl. Acad. Sci. U. S. A. 2003, 100, 10611-6.

(45) Chinnathambi, S.; Karthikeyan, S.; Velmurugan, D.; Hanagata, N.; Aruna, P.; Ganesan, S. 2015, 2015, 1-12.

(46) Sap, A.; DeZitter, E.; VanMeervelt, L.; Parac-Vogt, T. N. Chemistry A European Journal 2015, 21, 11692-11695.

(47) Jehle, S.; Vollmar, B. S.; Bardiaux, B.; Dove, K. K.; Rajagopal, P.; Gonen, T.; Oschkinat, H.; Klevit, R. E. Proceedings of the National Academy of Sciences 2011, 108, 6409-6414. 


\section{${ }_{364}$ Graphical TOC Entry}

Some journals require a graphical entry for the Table of Contents. This should be laid out "print ready" so that the sizing of the text is correct. Inside the tocentry environment, the font used is Helvetica $8 \mathrm{pt}$, as required by Journal of the American Chemical Society.

The surrounding frame is $9 \mathrm{~cm}$ by $3.5 \mathrm{~cm}$, which is the maximum permitted for Journal of the American Chemical Society graphical table of content entries. The box will not resize if the content is too big: instead it will overflow the edge of the box.

This box and the associated title will always be printed on a separate page at the end of the document. 


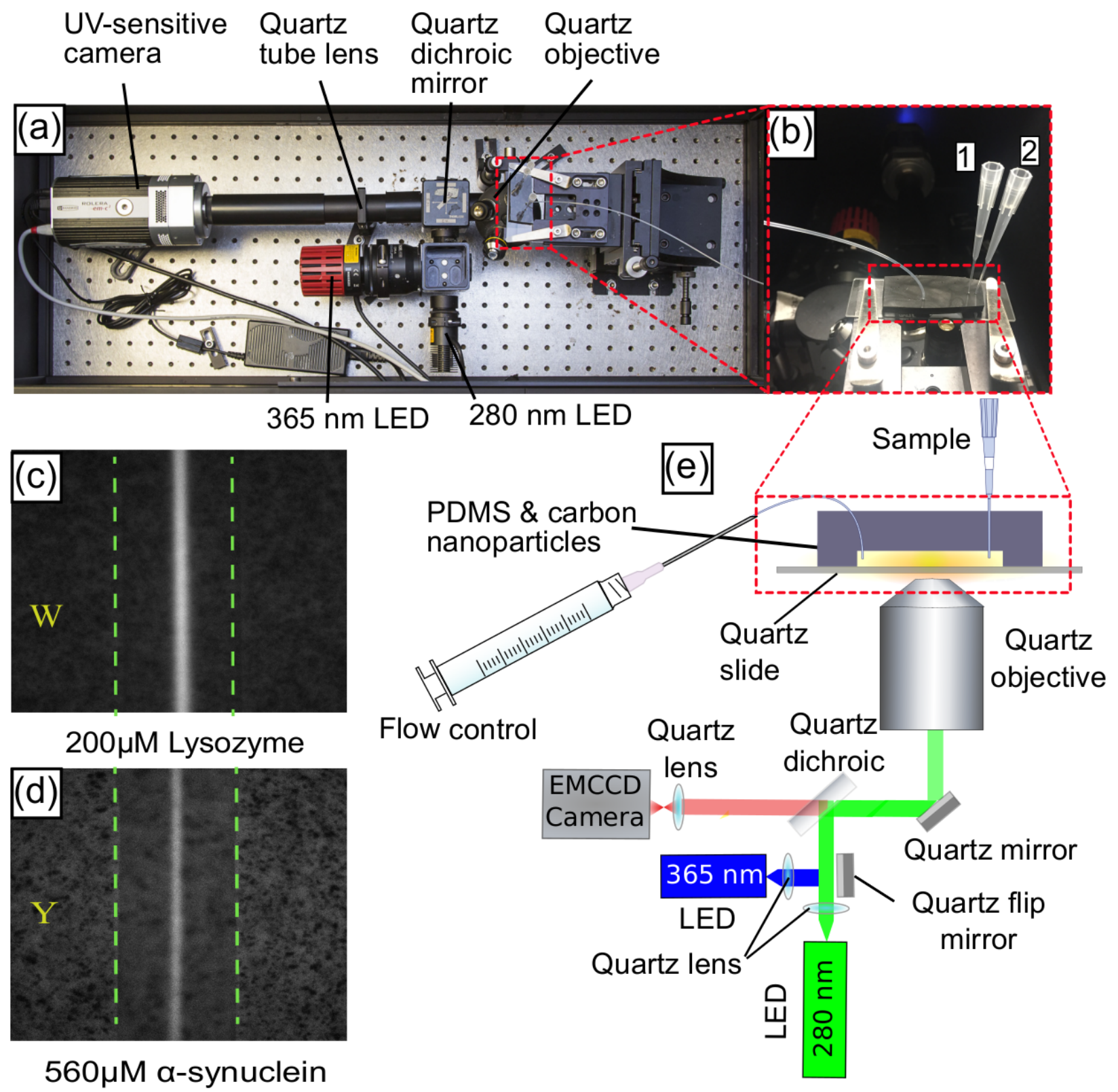

Figure 1: Deep-UV LED fluorescence platform to detect and quantify the intrinsic fluorescence from proteins in microfluidic systems, a. Photograph of the experimental set-up. b. A microfluidic device bonded to a quartz slide is placed on the detection stage. The protein sample and buffer are flowed through inlets 1 and 2. c. Autofluorescence of $200 \mu \mathrm{M}$ Lysozyme from Tryptophan [W] emission, d. Autofluorescence of $560 \mu \mathrm{M} \alpha$-synuclein from Tyrosine $[Y]$ emission. e. Schematic illustration of the optical set-up. Green dotted lines in c and d denote the ends of the microfluidic channels, where protein sample only occupies the middle of the channel. 


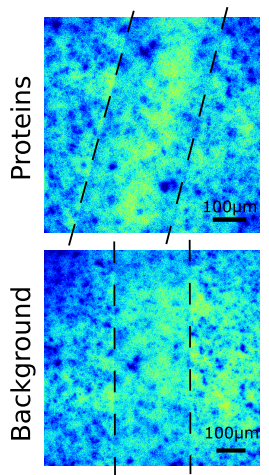

(a)

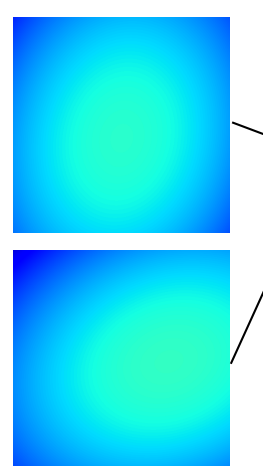

(b)

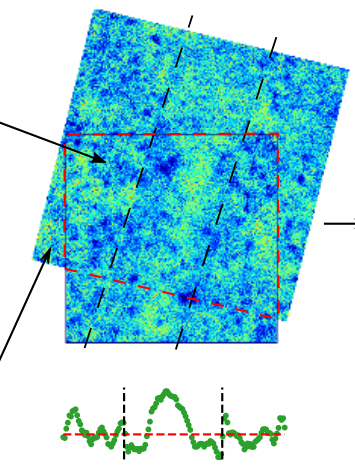

(c)

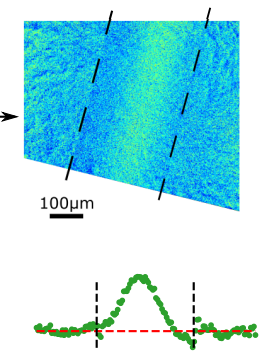

(d)

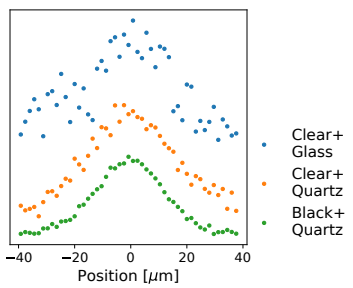

(e)

Figure 2: Quantitative background correction significantly improves signal to noise ratios of intrinsic fluorescence on chip. (a) Images taken with the CCD camera of both the channel containing protein BSA and of the background alone at position 12 (figure 3a). The tilt and scale difference is exaggerated to make the process easier to visualise. (b) First, the intensity is fit with a polynomial and the images are flattened, as can be seen when comparing (a) and (c). (c) Second, the difference in angle, scale, and $x-y$ offset is detected, resulting in an overlap between the two images. (d) As the images are flat and are overlapping, they can be subtracted to extract the relevant data. The change in profile is outlined on the bottom of (c) and (d). The topological noise introduced by the black nano-powder is removed. (e) Profiles at position 0 (figure 3a) in the microfluidic diffusional sizing device. Three microfluidic sizing chips are compared: clear PDMS bonded to glass, clear PDMS bonded to quartz, and black PDMS bonded to quartz. 

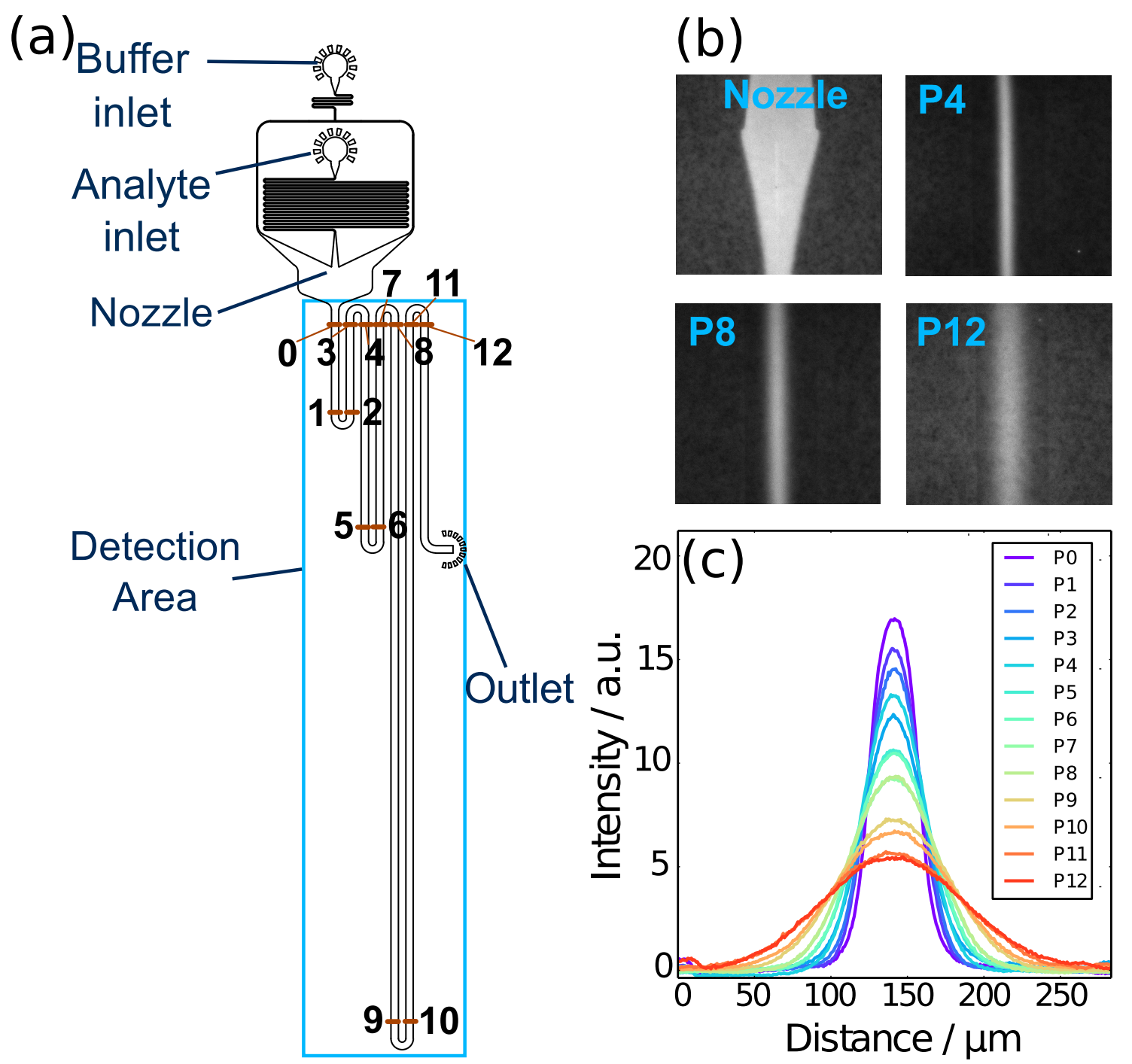

Figure 3: Overview of the microfluidic diffusional sizing measurements. (a) Channel geometry of the microfluidic diffusion device. The buffer and sample are loaded in their respective inlets and drawn through the device through the device with a syringe and pump connected to the outlet. Images of the lateral diffusion of sample into buffer are taken at positions 0-12 in the detection area. (b) Images of $15 \mu \mathrm{M}$ BSA taken at the nozzle and positions 4, 8 and 12. The extent of diffusion is greater further along the length of the diffusion channels. (c) Lateral scans of the imaged diffusion profiles in (b) from positions 0 through 12 . These profiles are fit to a linear combination of simulated basis functions in order to extract the sample's diffusion coefficient. 
(a)
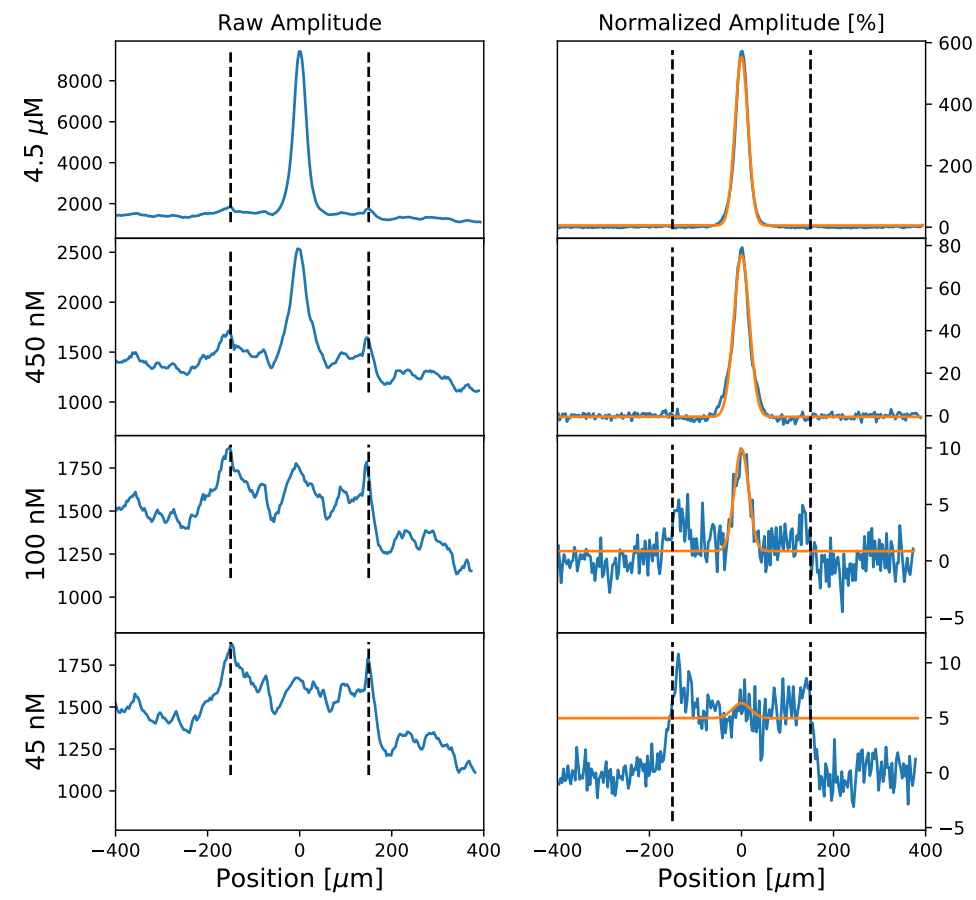

(b)

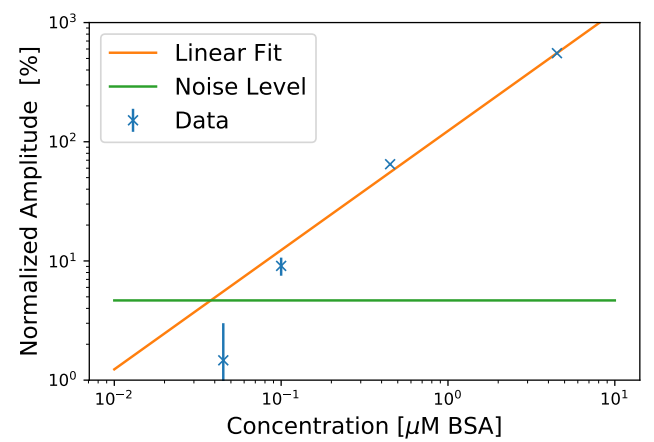

Figure 4: (a) Profiles at position 0 (figure 3a) of the diffusion device for different BSA concentrations. The positions of the channel walls are indicated by two dashed black lines. Left: raw profiles. Right: background-corrected profiles. The orange line on the corrected background corresponds to the Gaussian fit, and the amplitude of that fit is reported plotted in (b). The baseline in the channel $(-150$ to $150 \mu \mathrm{m})$ is slightly higher than outside the channel. (b) Amplitude of the profile plotted against BSA concentration on a log-log scale. The lowest detected amplitude is $100 \mathrm{nM}$ BSA. Representative profiles are shown in (a). The errors bars correspond to the standard deviation of the noise, and the green line correspond to 3 standard deviation of the noise. 


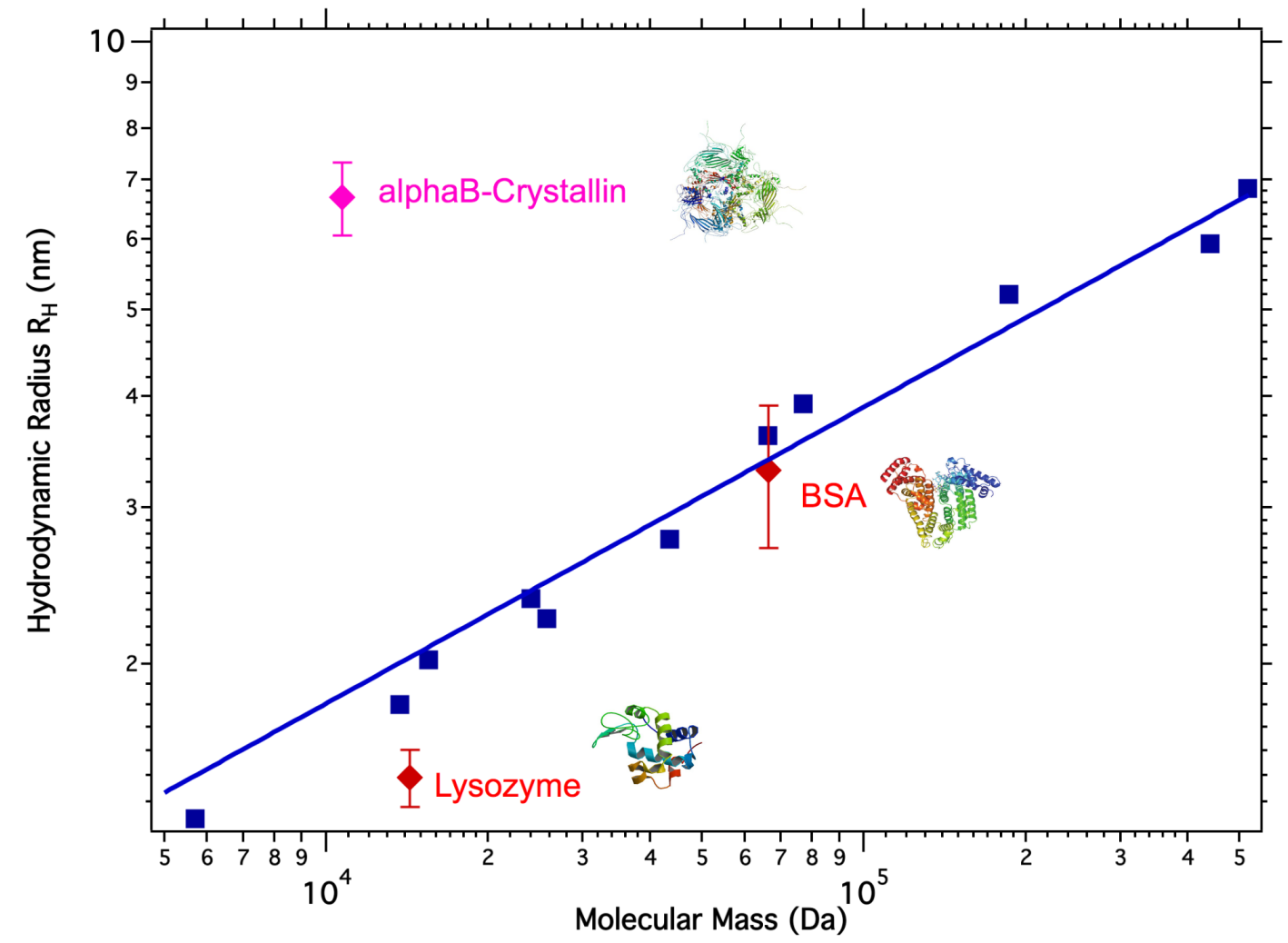

Figure 5: Blue squares are the hydrodynamic radii of different monomeric proteins plotted as a function of their molecular mass from reference ${ }^{40,41}$ and the blue solid line is the corresponding fit. Red data points are of BSA and lysozyme monomers, and the pink data point corresponds to alphaB-crystallin obtained using our platform. The size of alphaB-crystallin deviates significantly from the expected hydrodynamic radius calculated from its monomeric molecular weight, and thus the measured protein size provides strong solution-phase evidence of protein complex formation under native conditions. The structure of the proteins ${ }^{45-47}$ is shown next to their corresponding data point Error bars denote the standard deviation of triplicate measurements repeated in separately fabricated devices or under different flow rates. 